\title{
Polarization Sensitive Optical Coherence Tomography with Spectroscopic Analysis
}

\author{
M. Strąkowski*, J. Pluciński, and B.B. Kosmowski \\ Department of Optoelectronics and Electronic Systems, Faculty of Electronics, Telecommunications and Informatics \\ Gdańsk University of Technology, G. Narutowicza 11/12, 80-233 Gdańsk, Poland
}

\begin{abstract}
The optical coherence tomography is an optical measurement technique used for investigation of wide range of scattering or semitransparent materials. The optical coherence tomography enables surface and subsurface examination of different types of materials to be performed in non-contact and non-destructive way. Our research have been concentrated on optical coherence tomography systems with polarization sensitive analysis. Such a combination of optical measurements methods improves optical coherence tomography visualization contrast and also delivers some extra information about investigated devices. However, applying polarization sensitive analysis is associated with problems related to the use of ultra broadband optical sources. We have managed studies on the improvement of signal processing method. According to obtained results the problem of signal processing method in polarization sensitive optical coherence tomography can be solved by the use of the spectroscopic analysis. In this paper we present a brief discussion about the polarization sensitive optical coherence tomography measurements supported by spectroscopic analysis of backscattered light from investigated device.
\end{abstract}

PACS: 42.79.-e, 42.87.-d, 42.79.Sz, 42.79.Ta

\section{Introduction}

Non-invasive measurement methods are a versatile tool employed in materials science, manufacturing process control, and quality control. Moreover, they can be used for real-time monitoring of a broad range of devices. Our research has been concentrated on optical coherence tomography (OCT) for technical materials examination. OCT is a unique technique of cross-sectional and three-dimensional visualization of inner structure of different types of materials and devices. This method enables in situ investigation in non-contact and non-destructive way. Using OCT one can analyze the depth structure of investigated materials with measurement resolution of a few $\mu \mathrm{m}$, high sensitivity and high dynamic range $[1,2]$. Nowadays, the advantages of OCT make it applied in medical diagnosis, especially in ophthalmology, dermatology, stomatology, endoscopy $[3,4]$ and also in industry and science. Apart from medical applications, the OCT is used for material characterization, surface and subsurface defect detection, strain fields mapping in polymers, ceramic materials examination [5-8], as well as art conservation [9].

The OCT is based on the interference of the light beams, which have a short temporal coherence (white light interferometry (WLI) or low-coherence interferom-

\footnotetext{
* corresponding author; e-mail:

marcin.strakowski@eti.pg.gda.pl
}

etry (LCI)). Thereby the light backscattered from the particular scattering points inside the investigated object can be selectively detected, which is needed for tomography imaging $[1,2,10-16]$. There are several types of OCT systems $[7,11]$. The main classification of OCT systems is performed due to the measurement signal processing.

Till now there are three generations of OCT systems, which show the progress in this branch. The first one is the time domain OCT (TD-OCT). The measurement signal is processing in time domain, therefore, the TD-OCT systems need some mechanical parts for tuning the main interferometer. This causes low imaging frame rate increasing measurement time. The next generation belongs to spectral domain OCT (SD-OCT) systems. The interference signal is recorded by optical spectrometer. Usually the detection system is made of optical diffraction grating and an array of detectors. The signal processing is performed in frequency domain, which brings all information about scattering points placed on light beam direction inside tested device. The SD-OCT does not need any mechanical parts, which has to be used in TD-OCT. Therefore the SD-OCT systems are very fast and enable materials structure visualization in real time. The last generation of OCT is swept source OCT systems (SS-OCT). The measurement signal processing is performed in frequency domain, however, the ultrafast tunable laser and single detector have been used instead of broadband light source and the matrix of detectors, respectively. Relating to the SD-OCT, it increases imaging frame rate, signal to noise ratio and also measurement 
dynamic level.

Besides of conventional OCT systems, which deliver only information about inner structure of tested device, there are also OCT systems with additional functionality. The main extensions make the OCT Doppler analysis possible (D-OCT) [3] or polarization sensitive (PS-OCT) $[2,5,6,8]$.

Our research interests have been concentrated on OCT with polarization sensitive analysis (PS-OCT). PS-OCT is an extension of OCT which delivers extra information about optical anisotropy changes inside the structure, making it a useful tool for inspection and characterization of a broad range of materials $[2,7,11]$. Polarization sensitive analysis provides unique benefits to OCT measurements, which allow studying phenomena occurring in tested device that cannot be investigated by conventional OCT methods [7]. However, currently available PS-OCT systems have significant measurement limitations associated with using ultra-broadband light sources and birefringent principles of the investigated devices. These problems have been described in the following sections, as well as PS-OCT features.

\section{Polarization measurements problems in OCT systems}

Contrary to conventional OCT systems, PS-OCT needs stable polarization state of the light guided through the low-coherence interferometer to be controlled precisely. For polarization sensitive analysis the polarization diversity detection must be applied. An example of the PS-OCT has been presented in Fig. 1.

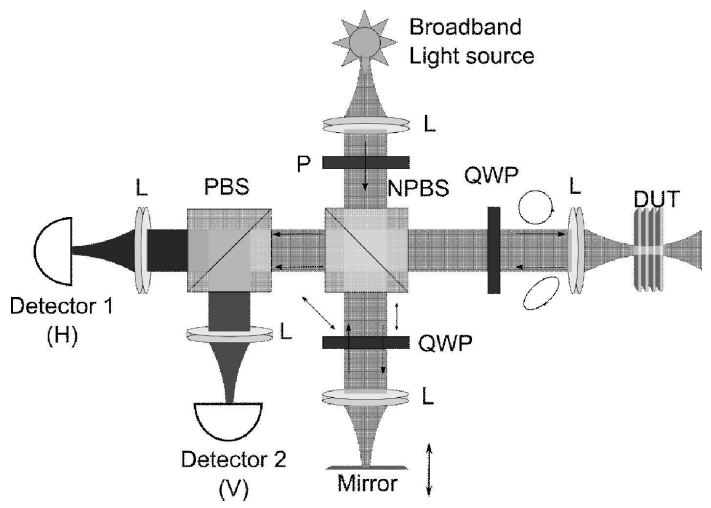

Fig. 1. PS-OCT system setup.

The PS-OCT system, presented in Fig. 1, is based on low-coherent interferometer in the Michelson configuration. The interferometer is illuminated by a broadband light source. The light beam from the source is vertically polarized by linear polarizer $(\mathrm{P})$ and directed to the measurement and the reference arms of the interferometer by the beamsplitter (NPBS). The polarization state of light propagating in measurement arm is forced to circular state and, then, the beam is focused inside the structure of the tested device. The light backscattered from measurement arm and backreflected from reference arm are recombined and subsequently divide into two components having orthogonal polarization states. One of the interference signals, which has a linear horizontal polarization state, is recorded by the detector $1(\mathrm{H})$ and the other one is recorded by detector $2(\mathrm{~V})$. Polarization sensitive analysis is based on the relationship of the amplitudes and phases of the recorded signals. As the results, the depth profile of the retardation angle and the optical axis orientation of the device are obtained. These characteristics depict local changes of the device birefringence. The calculations have been described by Eqs. (1) and (2):

$$
\begin{aligned}
& \Gamma(z)=a \tan \left(\frac{\left|I_{\mathrm{H}}(z)\right|}{\left|I_{\mathrm{V}}(z)\right|}\right), \\
& \Theta(z)=\frac{\pi-\Delta \varphi(z)}{2},
\end{aligned}
$$

where $\Gamma(z)$ - depth $(z)$ profile of the retardation angle changes inside tested device, $\Theta(z)$ - depth profile of the optical axis orientation of the device, $I_{\mathrm{H}}(z)$ and $I_{\mathrm{V}}(z)-$ recorded interference signals at detector 1 and detector 2 , respectively, $\Delta \varphi(z)$ - phase difference between recorded interference signals $[1,5-7,11]$.

According to the Jones formalism the $\Gamma(z)$ and $\Theta(z)$ describe the birefringence of the device completely. However, its polarization properties are determined for central wavelength of the broadband light source, which may cause a significant measurement error. Our studies on ultrahigh resolution PS-OCT systems show the relationship between the measurement accuracy of the polarization parameters and the spectral width of the light source. During our research we had made a numerical model of the PS-OCT system, which allowed us to investigate this problem. The obtained results permit us to conclude that if the broadband light source does not fulfill the relation

$$
\lambda_{0} \gg \Delta \lambda,
$$

where $\lambda_{0}$ - central wavelength and $\Delta \lambda-$ spectral width of the light source, the influence of the light spectral width on measurement accuracy of the polarization parameters cannot be neglected [12]. Summarized results of the simulations have been presented in Fig. 2.

The chart in Fig. 2 shows the simulation results as a relationship between uncertainty of measurement polarization quantity and the retardation angle difference $\Delta \Gamma$. The $\Delta \Gamma$ is calculated as the difference between extreme wavelengths of the spectral width of the light source. From the simulation results the measurement error of the $\Theta(z)$ and $\Delta \Gamma(z)$ characteristics may reach about $20 \%$ of maximum quantity value. The problem becomes very significant if the ultra-broadband source like supercontinuum is used. Therefore, the new method of measurement signal processing should be developed for ultrahigh resolution PS OCT systems. From our research and studies we propose novel application of spectroscopic analysis based on the Fourier transform principles. This is new 


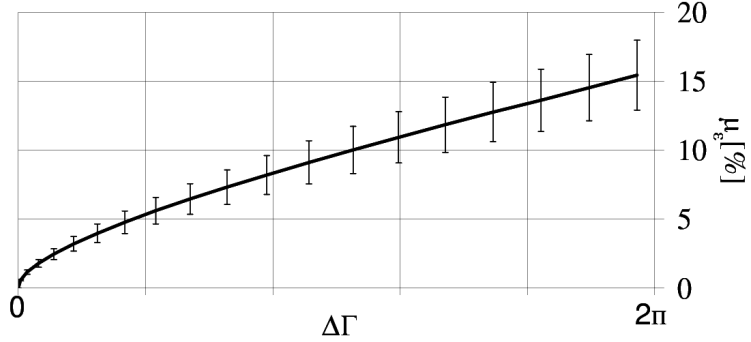

Fig. 2. Simulation results of the PS-OCT measurement error. $\Delta \Gamma$ - retardation angle difference over the spectral width of the light source, $\mu_{\varepsilon}$ - uncertainty related to maximum value of measured quantity.

approach in PS-OCT, which enables the polarization sensitive analysis to be performed over the spectral range of the broadband light source.

\section{Spectroscopic analysis for PS-OCT}

Spectroscopic analysis for PS-OCT delivers the polarization quantities, which are determined on the basis of the spectral characteristic of the light, which is backscattered from the particular points inside investigated device. One of the most important problems, which must be solved, is the selective reconstruction of the spectral characteristics from recorded interference signals. Consider the equation

$$
\begin{aligned}
& S_{\mathrm{D}}(k)=(\mathrm{DC})+2 \sum_{n=1}^{N}\left|S_{R n}(k)\right| \cos \left(2 k\left(z_{\mathrm{R}}-z_{n}\right)\right. \\
& \left.\quad+\arg \left(S_{R n}(k)\right)\right), \\
& \quad+(\text { autocorrelation terms }),
\end{aligned}
$$

which describes the OCT measurement signal in spectral domain. The $S_{R n}(k)$ is the cross-spectral density of the interfering beams. It depends on the spectral characteristics of the light from the measurement and reference arm of the low-coherent interferometer. By the use of the Fourier transform (FT) it is possible to transfer the $S_{\mathrm{D}}(k)$ function to time domain, which has been shown as the relation $(5)$ :

$$
\begin{aligned}
& \mathrm{FT}\left\{S_{\mathrm{D}}(k)\right\}=I_{\mathrm{D}}(z)=(\mathrm{DC} \text { terms }) \\
& \quad+\sum_{n=1}^{N} G_{R n}(z) \otimes\left(\delta\left(z \pm 2\left(z_{\mathrm{R}}-z_{n}\right)\right)\right) \\
& \quad+(\text { autocorrelation terms }),
\end{aligned}
$$

where $G_{R n}(z)$ - cross-correlation function of the light from reference arm and backscattered from particular $n$ point inside the investigated sample, $z_{\mathrm{R}}-z_{n}$ determines the optical delay between interfering beams. The cross correlation function $G_{R n}(z)$ and cross spectral density $S_{R n}(k)$ are related to each other by the pair of the Fourier transforms. Therefore, if the $G_{R n}(z)$ function can be obtained, the $S_{R n}(k)$ will be determined. According to (5), the $G_{R n}(z)$ function is separated in time domain, which facilitates its extraction from $I_{\mathrm{D}}(z)$ characteristic. Based on the described relations the measurement signal procedure can be developed in order to obtain the complex spectral characteristic of the backscattered light [13-15]. An example of the signal processing, which has been described in detail in $[13,14]$, has been shown in Fig. 3.

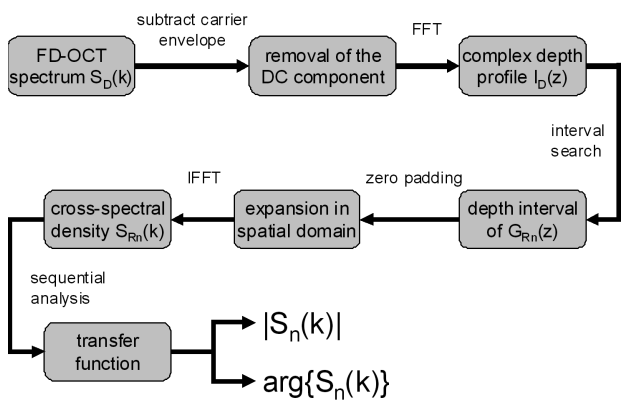

Fig. 3. Signal processing in OCT with spectroscopic analysis.

The final result of the presented procedure (Fig. 3) is the complex function $S_{n}(k)$. The $S_{n}(k)$ is the spectral density of light, which is backscattered from particular $n$ point inside the investigated device. Applying this method to PS-OCT the $S_{n}(k)$ functions can be determined for both orthogonal polarization states of the interference signal. This enables to provide a polarization sensitive analysis over the spectral range of the broadband light source. This method has been applied to our numerical model and the simulations have been carried out. The simulations results allow us to assess the usefulness of the method and compare it to the conventional approach. The simulation results for conventional PS-OCT and PS-OCT with spectroscopic analysis (PS-SC-OCT) have been summarized in the graph in Fig. 4.

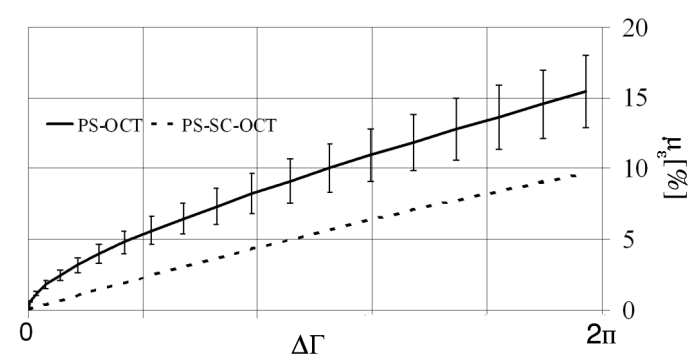

Fig. 4. Simulation results of the PS-OCT and PS-SC-OCT measurement error. $\Delta \Gamma-$ retardation angle difference over the spectral width of the light source, $\mu_{\varepsilon}$ - uncertainty related to maximum value of measured quantity, PS-OCT - simulation data obtained for the model of conventional PS-OCT, PS-SC-OCT - simulation data obtained for the model of PS-OCT with spectroscopic analysis.

The plot in Fig. 4 presents the simulation of measurement uncertainty as a function of the retardation angle 
difference over the light source spectrum. Applying the spectroscopic analysis reduced the measurement uncertainty to value under $10 \%$.

\section{Conclusions}

During our research and studies we have investigated a new application of spectroscopic analysis for PS-OCT. According to obtained results this method can improve the polarization sensitive analysis by reducing the measurement error. Concluding, the spectroscopic analysis can be a useful method in polarization sensitive optical coherence tomography. It brings a unique benefits to PS-OCT measurement delivering full spectral information about backscattered light from the particular points inside the tested device. It enables an investigation of optical anisotropy changes occurring inside the device in the range of broadband light source spectrum. Moreover, combination of PS-OCT and spectroscopic analysis enables to conduct optical anisotropy and spectroscopic measurements simultaneously. Therefore, the polarization sensitive optical coherence tomography with spectroscopic analysis can be interesting tool for the investigation and quality assessment of the complex objects like printed electronics parts or polymer composites materials.

\section{Acknowledgments}

This study was partially supported by the Polish Ministry of Science and Higher Education under the grant No. N N515 335636, and DS programs of the Faculty of Electronics, Telecommunications and Informatics, Gdańsk University of Technology.

\section{References}

[1] A.F. Fercher, W. Drexler, C.K. Hitzenberg, Rep. Prog. Phys. 66, 239 (2003).

[2] M. Strąkowski, J. Pluciński, et al., Sensors Actuators A 142, 104 (2008).

[3] A. Szkulmowska, M. Szkulmowski, A. Kowalczyk, M. Wojtkowski, Opt. Lett. 33, 1425 (2008).

[4] M. Wojtkowski, T. Bajraszewski, P. Targowski, A. Kowalczyk, Opt. Lett. 28, 1745 (2003).

[5] D. Stifter, P. Burgholzer, O. Höglinger, E. Götzinger, C.K. Hitzenberger, Appl. Phys. 76, 947 (2003).

[6] D. Stifter, Appl. Phys. B 88, 337 (2007).

[7] M. Strąkowski, J. Pluciński, B.B. Kosmowski, Photon. Lett. Poland 1, 52 (2009).

[8] K. Wiesauer, M. Pirchen, E. Gotzinger, C.K. Hitzenberger, Composit. Sci. Technol. 67, 3051 (2007).

[9] P. Targowski, B. Rouba, M. Góra, L. Tymańska-Widmer, J. Marczak, A. Kowalczyk, Appl. Phys. A 92, 1 (2008).

[10] P. Hlubina, D. Ciprian, R. Chlebus, Opt. Commun. 281, 4008 (2008).

[11] J. Pluciński, R. Hypszer, P. Wierzba, M. Strąkowski, M. Jędrzejewska-Szczerska, M. Maciejewski, B.B. Kosmowski, Bull. Pol. Acad. Sci. - Techn. Sci. 56, 155 (2008).

[12] M. Strąkowski, Ph.D. Thesis, WETI PG 2010 (in Polish).

[13] Ch. Kasseck, V. Jaedicke, N.C. Gerhardt, H. Welp, M.R. Hofmann, Opt. Commun. 283, 4816 (2010).

[14] Ch. Kasseck, V. Jaedicke, N.C. Gerhardt, H. Welp, M.R. Hofmann, Proc. SPIE 7554, 75542T (2010).

[15] G. Latour, J. Moreau, M. Elias, J.-M. Frigerio, Opt. Commun. 283, 4810 (2010).

[16] M. Jędrzejewska-Szczerska, Europ. Phys. J. Spec. Top. 144, 230 (2007). 